\title{
Caracterización mineralógica y geoquímica de minerales hidratados de ambientes subterráneos: implicaciones para la exploración planetaria
}

\author{
Mineralogical and geochemical characterization of hydrated \\ minerals from subterranean environments: implications for \\ planetary exploration
}

F. Gázquez ${ }^{1}$, F. Rull ${ }^{2}$, J.M. Calaforra ${ }^{3}$, G. Venegas ${ }^{2}$, J.A. Manrique ${ }^{2}$, A. Sanz ${ }^{2}$, J. Medina $^{2}$, A. Catalá-Espí ${ }^{2}$, A. Sansano ${ }^{2}$, R. Navarro ${ }^{2}$, P. Forti ${ }^{4}$, J. De Waele ${ }^{4}$, J. Martínez-Frías ${ }^{2,5}$

1 Godwin Laboratory for Palaeoclimate Research, Dept. of Earth Sciences. University of Cambridge, CB1 1DS Cambridge, Reino Unido. Email: fg331@cam.ac.uk

2 Unidad Asociada UVA-CSIC al Centro de Astrobiología (ERICA), Universidad de Valladolid, Parque Tecnológico Boecillo, 47151, Valladolid, España

3 Grupos de Recursos hídricos y Geología ambiental, Universidad de Almería, Crt. Sacramento s/n. La Cañada de San Urbano, 04120, Almería, España

4 Italian Institute of Speleology, Department of Biological, Geological and Environmental Sciences, University of Bologna. Via Zamboni, 67, 40126. Bologna, Italia

5 Instituto de Geociencia, IGEO (CSIC-UCM), Facultad de Ciencias Geológicas C/ José Antonio Novais 2, Ciudad Universitaria, 28040 Madrid, España

\section{RESUMEN}

El reciente descubrimiento de minerales hidratados sobre la superficie de Marte sugiere la presencia de importantes cantidades de agua líquida durante algunas etapas de su historia geológica. A raíz de este hallazgo, los estudios sobre minerales hidratados en ambientes terrestres como potenciales análogos marcianos han adquirido gran relevancia. En el presente trabajo se han estudiado las características mineralógicas y geoquímicas de minerales hidratados procedentes de varias cuevas y minas españolas y de la región minera de Iglesias-Carbonia (Cerdeña, Italia) mediante técnicas espectroscópicas implicadas en misiones de exploración marciana presentes y futuras, con el fin de evaluar su potencial para la detección de este tipo de minerales. Por un lado, se ha utilizado la espectroscopia Raman, que formará parte de la carga científica de la misión ExoMars de la Agencia Espacial Europea. Por otro lado, la espectroscopia de IR, otra de las técnicas involucradas en esta misión de la ESA, así como la espectroscopia LIBS y la combinación de difracción-fluorescencia de rayos X (DRX-FRX), ambas a bordo de la misión MSL de la NASA. Estas técnicas han permitido identificar sulfatos (yeso, epsomita, jarosita y glaucocerinita), silicatos (hemimorfita) y carbonatos (hidrocincita e hidromagnesita), todos ellos minerales hidratados y algunos de los cuales también han sido descritos en Marte. Por otro lado, se han abordado los procesos de formación de estos minerales y las potenciales analogías con la mineralogénesis en Marte. Del conjunto de técnicas empleadas, la combinación Raman-LIBS se perfila como la opción más eficiente para la detección de minerales hidratados en condiciones marcianas.

Palabras clave: minerales hidratados; ExoMars; espectroscopia Raman; LIBS; Marte; análogos marcianos

Recibido: 18 de febrero de 2014 / Aceptado: 30 de abril de 2014 / Publicado: 25 de agosto de 2014

Citation / Cómo citar este artículo: Gázquez, F. et al. (2014) Caracterización mineralógica y geoquímica de minerales hidratados de ambientes subterráneos: implicaciones para la exploración planetaria. Estudios Geológicos 70(2): e009. doi: http://dx.doi.org/10.3989/ egeol.41688.314.

Copyright: (c) 2014 CSIC. This is an open-access article distributed under the terms of the Creative Commons Attribution-Non Commercial (by-nc) Spain 3.0 License. 


\section{ABSTRACT}

The recent discovery of hydrated sulfates on the Martian surface suggests that widespread wet conditions were present during its early geological history. Upon this discovery, a growing interest has emerged in the study of this group of minerals from terrestrial environments as potential Martian analogs. Here, we evaluate the potential of various analytical techniques involved in current and future mission to Mars for detecting hydrated minerals from caves and mines of Spain and the mining district of Iglesias-Carbonia (Sardinia, Italy). Minerals were analyzed by Raman spectroscopy, which will be included in the payload of the ESA's 2018 ExoMars mission. On the other hand, IR spectroscopy, also included in the ExoMars mission, as well as LIBS spectroscopy and a combined XRD-XRF analyzer, both onboard the Curiosity rover of NASA's MSL mission, were utilized. Hydrated sulfates (gypsum, epsomite, jarosite and glaucocerinite), silicates (hemimorphite) and carbonates (hydrozincite and hydromagnesite) were characterized. Most of these minerals have also been detected on the Martian surface. The mechanisms involved in the genesis of these minerals and the potential analogies with the minerogenesis on Mars are discussed. The Raman-LIBS combination appears to be the most powerful tool for detecting hydrated minerals in Martian conditions. This technology will probably be considered to be onboard of further planetary missions.

Keywords: hydrated minerals; ExoMars; Raman spectroscopy; LIBS; Mars exploration; mine minerals

\section{Introducción}

El reciente descubrimiento de minerales hidratados sobre la superficie de Marte ha generado un enorme interés sobre las características mineralógicas y genéticas de este grupo de minerales en la Tierra. En particular, los estudios relacionados con la mineralogía y geoquímica de sulfatos hidratados como potenciales análogos de Marte han adquirido gran relevancia durante la última década (Martínez-Frías et al., 2004; 2006; Baioni et al., 2009; Ling \& Wang, 2010; Szynkiewicz et al., 2010; entre otros).

Las primeras evidencias que apuntaban a la presencia de depósitos masivos de sulfatos de magnesio y calcio hidratados en Marte fueron proporcionadas por el espectrómetro OMEGA de la sonda Mars Express y el CRISM del Mars Reconnaissance Orbiter (MRO) (Arvidson et al., 2005; Bibring et al., 2005; Gendrin et al., 2005; Langevin et al., 2005). Hasta la fecha, minerales como la bassanita $\left(\mathrm{CaSO}_{4} \cdot \sim 1 / 2 \mathrm{H}_{2} \mathrm{O}\right)$ y la kieserita $\left(\mathrm{MgSO}_{4} \cdot \mathrm{H}_{2} \mathrm{O}\right)$ han sido identificados en Marte (Gendrin et al., 2005; Zhu et al., 2006; Wray et al., 2010), además de la posible presencia de starkeyita $\left(\mathrm{MgSO}_{4} \cdot 4 \mathrm{H}_{2} \mathrm{O}\right)$, pentahidrita $\left(\mathrm{MgSO}_{4} \cdot 5 \mathrm{H}_{2} \mathrm{O}\right)$, hexahidrita $\left(\mathrm{MgSO}_{4} \cdot 6 \mathrm{H}_{2} \mathrm{O}\right)$, epsomita $\left(\mathrm{MgSO}_{4} \cdot 7 \mathrm{H}_{2} \mathrm{O}\right)$, meridianita $\left(\mathrm{MgSO}_{4} \cdot 11 \mathrm{H}_{2} \mathrm{O}\right)$, szomolnokita $\left(\mathrm{Fe}^{2+} \mathrm{SO}_{4} \cdot \mathrm{H}_{2} \mathrm{O}\right)$ y ferricopiapita $\left(\mathrm{Fe}^{3+}{ }_{2 / 3} \mathrm{Fe}^{3+}{ }_{4}\left(\mathrm{SO}_{4}\right)_{6}(\mathrm{OH})_{2} \cdot 20 \mathrm{H}_{2} \mathrm{O}\right)$ (Lane et al., 2004; Wilson \& Bish, 2012).

Estos orbitadores apenas detectaron sulfatos de hierro sobre la superficie del planeta rojo (Milliken et al., 2007; Wiseman et al., 2008), lo que contrasta con los resultados obtenidos por el espectrómetro Mössbauer MIMOS II incluido en el rover Opportunity de la NASA, que sugiere la presencia generalizada de jarosita $\left(\mathrm{KFe}^{3+}\left(\mathrm{SO}_{4}\right)_{2}(\mathrm{OH})_{6}\right)$ a lo largo de un trayecto de $11 \mathrm{~km}$ en el Meridiani Planum de Marte (Klingelhöfer et al., 2004; Squyres et al., 2004). Por otro lado, el sulfato de calcio (probablemente yeso, $\mathrm{CaSO}_{4} \cdot 2 \mathrm{H}_{2} \mathrm{O}$ ) ha sido recientemente identificado en torno al Polo Norte marciano, formando parte de un denso campo de dunas constituidas por arenas yesíferas (Massé et al., 2012). Además, el rover Opportunity encontró en 2011 venas minerales de color claro identificadas como yeso en base a su aspecto y composición química, cuyo origen se ha relacionado con procesos hidrotermales (Showstack, 2011).

Los descubrimientos relacionados con minerales hidratados de naturaleza carbonática en Marte se reducen hasta la fecha a la detección de carbonatos de magnesio hidratado, probablemente hidromagnesita $\left(\mathrm{Mg}_{5}\left(\mathrm{CO}_{3}\right)_{4}(\mathrm{OH})_{2} \cdot 4 \mathrm{H}_{2} \mathrm{O}\right)$ y artinita $\left(\mathrm{Mg}_{2}\left(\mathrm{CO}_{3}\right)\right.$ $\left.(\mathrm{OH})_{2} \cdot 3 \mathrm{H}_{2} \mathrm{O}\right)($ Calvin et al., 1994). Silicatos hidratados, principalmente filosilicatos, también han sido descritos sobre la superficie de Marte, cuyo origen se debería a la alteración del regolito basáltico en condiciones acuosas y probablemente no tan ácidas como las requeridas para la formación de otros minerales (Mustard et al., 2008), como pudo ser el caso de la jarosita (Squyres et al., 2004; Zolotov \& Shock, 2005).

Si se tiene en cuenta que la mayoría de minerales hidratados terrestres tienen su origen en procesos íntimamente ligados a la presencia de agua líquida, 
las investigaciones centradas en estos materiales son de extrema importancia para entender la génesis de los minerales hidratados marcianos y su relación con posibles signos de vida pasada o nichos de vida presente en el planeta.

Desde la pasada década, se han empezado a plantear los ambientes subterráneos terrestres como posibles análogos marcianos (Boston et al., 2001). La ausencia de radiación solar, la temperatura prácticamente constante a escala diaria y estacional, así como la presencia de agua líquida son algunos de los elementos que hacen atractivos los ambientes subterráneos para la investigación planetaria y astrobiológica. Esta línea de investigación ha adquirido mayor relevancia a partir del reciente descubrimiento de lo que podrían ser entradas de cuevas y tubos volcánicos en la superficie de Marte (Baioni et al., 2009; Cushing, 2012). El interés suscitado por estos ambientes radica tanto en la hipotética presencia de biomarcadores en el subsuelo y en las cuevas de Marte, como en el potencial que podrían presentar las cavidades marcianas como base de futuras misiones tripuladas al planeta rojo (Boston et al., 2003; Kisdi \& Tatnall, 2011).

En el presente trabajo se han estudiado las características mineralógicas y geoquímicas de minerales hidratados procedentes de la Mina Rica del Pilar de Jaravía en Pulpí (Almería, España), la Cueva de El Soplao (Cantabria) y varias cuevas y minas de la región sudoccidental de Cerdeña (Italia). Procesos de mineralogénesis hidrotermales, alteraciones de materiales preexistentes en condiciones ácidas y mecanismos de intensa evaporación ocurridos en estas cavidades son discutidos en el presente trabajo, así como sus posibles analogías con los procesos de formación de minerales hidratados en Marte.

Los análisis químicos y mineralógicos se han llevado a cabo con técnicas implicadas en misiones planetarias presentes y futuras, en algunos casos usando prototipos de instrumentos especialmente diseñados para formar parte de estas misiones. Éste es el caso del instrumento Raman e infrarrojo (IR), incluidos en la misión ExoMars de la ESA cuyo lanzamiento está previsto para 2018 (Rull \& MartínezFrías, 2006; López-Reyes et al., 2013), así como el equipo de análisis combinado de difracción (DRX) y fluorescencia (FRX) de rayos X, que es un prototipo gemelo del instrumento CheMin de la misión
MSL de la NASA (Bish et al., 2013; Blake et al., 2013). La técnica LIBS (Laser Induced Breakdown Spectroscopy), incluida en el instrumento ChemCam de la misión MSL (Lanza et al., 2010), también ha sido empleada para la caracterización geoquímica de estos minerales.

\section{Procedencia y características de las muestras}

\section{Mina Rica de Pulpí (yeso, epsomita y jarosita)}

El yeso estudiado en este trabajo procede de la Geoda gigante de Pulpí, ubicada en el interior de la Mina Rica de Pilar de Jaravía (Pulpí, Almería). Esta cavidad es uno de los pocos ejemplos descritos de una cueva cuyas paredes, techo y suelo están totalmente cubiertos por cristales pinacoidales de yeso selenítico, lo que la convierten en un elemento geológico único (Calaforra et al., 2001; García-Guinea, 2002) (Fig. 1A). La geoda fue descubierta en 1999 y tiene un volumen aproximado de $10.7 \mathrm{~m}^{3}$, presenta $8 \mathrm{~m}$ de longitud, por $1.8 \mathrm{~m}$ de anchura y $1.7 \mathrm{~m}$ de altura (Fernández-Cortés et al., 2006). El tamaño medio de los cristales es de $0.5 \times 0.4 \times 0.3 \mathrm{~m}$ aunque aparecen algunas "vigas" de yeso de hasta $2 \mathrm{~m}$ de longitud.

La mina donde se encuentra la geoda fue abandonada en los años 70. De ella se extraía hierro, plomo y plata desde finales del siglo XIX. La geoda se ubica a 50 metros de profundidad y está encajada en dolomías triásicas que forman parte de la Sierra del Aguilón, y que a su vez están enmarcadas en el complejo Alpujárride (Calaforra et al., 2001). La secuencia carbonática del Paleozoico está ligeramente metamorfizada y yace bajo materiales metapelíticos triásicos de baja permeabilidad. Las mineralizaciones de la mina del Pilar de Jaravía están relacionadas con eventos volcánicos acontecidos durante el Mioceno que también se han observado en el entorno, como es el caso de la Sierra de Almagrera (Martínez-Frías, 1998), donde también aparecen mineralizaciones de sulfuros (pirita, calcopirita y bournonita), sulfatos (mayoritariamente yeso, celestina y barita) y carbonatos de hierro (siderita).

En la Mina Rica, además de los cristales de yeso que forman parte de la geoda gigante, aparecen geodas de menor tamaño así como cristales de yeso rellenando fracturas de la roca caja. Por motivos de 

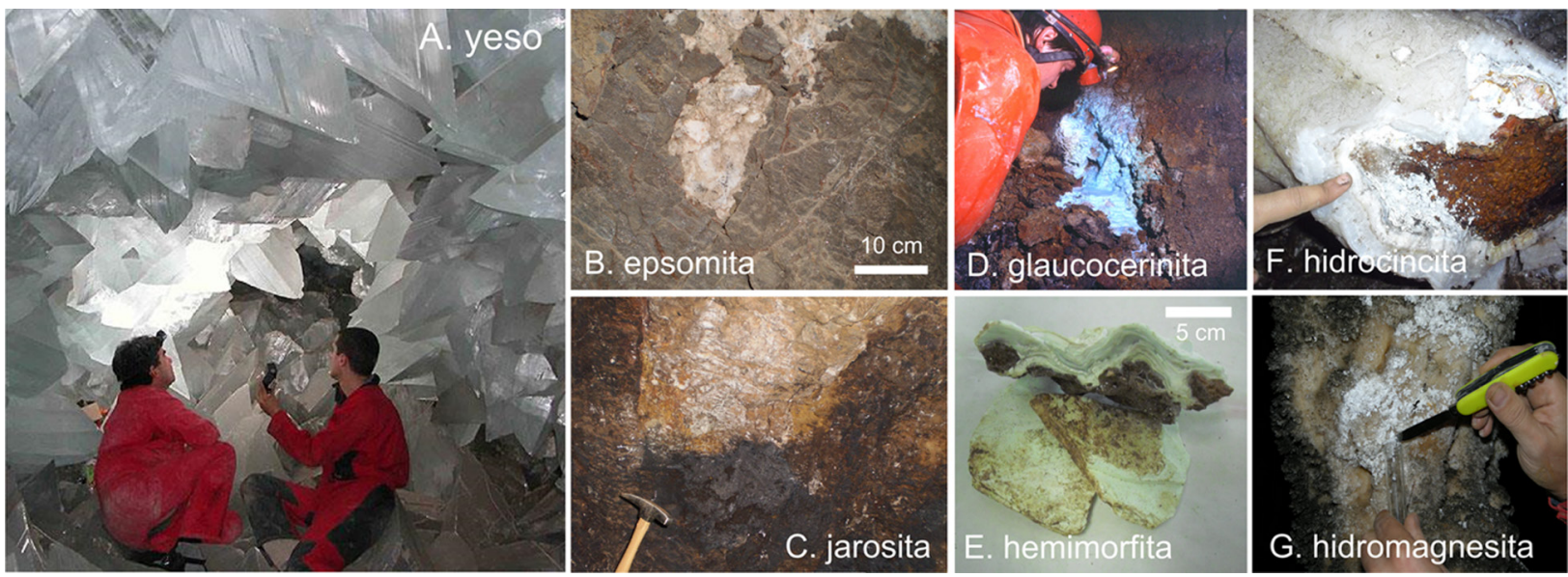

Fig. 1.-Muestras de minerales hidratados analizadas en este trabajo. A. Yeso. Geoda gigante de Pulpí (Mina Rica, Almería); B. Epsomita (Mina Rica, Almería); C. Jarosita (Mina Rica, Almería); D. Glaucocerinita (Mina Su Zurfuru, Cerdeña); E. Hemimorfita (Crovassa Quarziti, Cerdeña); F. Hidrocincita (Massa Riccardo, Cerdeña); G. Hidromagnesita (Cueva de El Soplao, Cantabria).

conservación, para los estudios llevados a cabo en este trabajo se tomó una muestra de una de estas geodas.

Además de yeso se han identificado hasta 27 minerales distintos mediante espectroscopia Raman (datos no publicados), de los cuales 7 son sulfatos hidratados entre los que destacan por su abundancia la epsomita y la jarosita. Los sulfatos de magnesio hidratados aparecen en forma de eflorescencias y costras pulverulentas sobre las paredes y techos de las galerías mineras, en particular en zonas cercanas a la entrada de la mina donde se producen intensas corrientes de aire. Una de estas costras fue recogida para su análisis en este estudio (Fig. 1B). Por otro lado, se tomó una muestra de jarosita que aparece en forma de venas de color ocre de hasta $30 \mathrm{~cm}$ de espesor y que se han observado fundamentalmente rellenando fracturas, así como entre estratos de filitas del miembro superior (Fig. 1C).

\section{Región minera Iglesias-Carbonia (glaucocerinita, hemimorfita e hidrocincita)}

La región sudoccidental de la isla de Cerdeña (Italia) ha sido objeto de actividades mineras durante los últimos 2.000 años, las cuales se intensificaron a partir del siglo XIX y prosiguieron de forma intensiva hasta los años 80 del siglo XX. La geología regional comprende areniscas metamorfizadas, filitas, dolomías y calizas karstificadas de edades comprendidas entre el Cámbrico inferior y el OrdovícicoSilúrico (Bechstadt \& Boni, 1996).

La mina Su Zurfuru está ubicada en el Distrito minero de Fluminimaggiore y fue objeto de actividad extractiva de sulfuros de $\mathrm{Zn}$ y $\mathrm{Pb}$ y fluorita $\left(\mathrm{CaF}_{2}\right)$ hasta los años 90. En sus galerías mineras aparecen formaciones de microgours que van desde colores blancos a azules intensos (Fig. 1D), particularmente en la zona denominada "Fuente del Zinc" (Forti et al., 2005; Gázquez et al., 2013a) aproximadamente a $500 \mathrm{~m}$ de la entrada principal de la mina. Las formaciones de microgours, sobre las cuales discurre un flujo laminar de agua, presentan hasta $3 \mathrm{~m}$ de longitud y $1.5 \mathrm{~m}$ de anchura, yacen sobre el suelo y en ocasiones sobre las paredes de las galerías. Gázquez et al., (2013a) encontraron glaucocerinita $\left((\mathrm{Zn}, \mathrm{Cu})_{5} \mathrm{AI}_{3}(\mathrm{OH})_{16}\left(\mathrm{SO}_{4}\right)_{1.5} \cdot 9 \mathrm{H}_{2} \mathrm{O}\right)$, yeso y fluorita en estas formaciones, que también han sido estudiadas en el presente trabajo mediante diversas técnicas.

Por otro lado, se ha analizado un fragmento de una colada de hemimorfita de más de $10 \mathrm{~m}$ de altura encontrada en la Crovassa Quarziti en el interior de la mina del Monte San Giovanni del distrito minero de Iglesias-Carbonia. Esta colada está constituida por láminas de hemimorfita de color azulado y verdoso que en muchas ocasiones aparecen depositadas sobre una base de óxidos de Fe y Mn (Fig. 1E) y en otras directamente sobre el sustrato cuarcítico, 
probablemente originado debido a procesos de silicificación de las dolomías cámbricas, como se ha observado en otras cuevas del entorno (Sauro et al., 2014). Se trata de una de las más de 50 cavidades hipogénicas descubiertas accidentalmente debido a la explotación de las mineralizaciones de sulfuros polimetálicos (galena y esfalerita) enriquecidos en plata en el Monte San Giovanni (Pagliara et al., 2010; Caddeo et al., 2011; De Waele et al, 2013; Gázquez et al., 2013b).

Las mineralizaciones de hidrocincita analizadas en este estudio proceden de la Cueva Massa Riccardo, interceptada por el frente de explotación minera de la mina del Monte San Giovanni. La hidrocincita se ha observado en forma de depósitos blancos laminados de pocos milímetros y depositados sobre mineralizaciones de óxidos de Fe y Mn y en muchas ocasiones cubiertos por cristalizaciones laminadas de calcita de hasta $30 \mathrm{~cm}$ de espesor (Fig. 1F).

\section{Cueva de El Soplao (hidromagnesita)}

La Cueva de El Soplao se ubica a 540 m s.n.m. en la Sierra de Arnero que a su vez se encuentra enmarcada en la Sierra del Escudo de Cabuérniga (Cantabria, Norte de España). La cueva se desarrolla en una unidad de carbonatos marinos de plataforma continental somera (Unidad de La Florida), de edad Aptiense. Además, la región tiene un tradicional interés metalogenético, puesto que las unidades carbonatadas que se estudian presentan grandes parches de dolomitización en los que se alojan importantes yacimientos minerales de plomo y zinc en la mina de La Florida y la propia cueva (Quesada et al., 2005). La Cueva de El Soplao, está abierta al público desde 2005 y es una de las cuevas turísticas españolas más visitadas y estudiadas desde el punto de vista científico (Gázquez et al., 2011, 2012a, 2014).

La hidromagnesita en El Soplao aparece formando parte de depósitos de moonmilk sobre otros espeleotemas y paredes en diversos puntos de la cavidad. En este trabajo se han estudiado muestras de hidromagnesita procedentes de la Galería de El Bosque, las cuales se han observado como agregados botroidales sobre estalagmitas. La disposición de los depósitos de moonmilk no es aleatoria sino que se localizan en un lateral de las estalagmitas sobre las que se desarrollan, claramente controlado por la dirección predominante del aire en la galería (Gázquez et al., 2010; 2012b).

\section{Métodos}

Los análisis geoquímicos y mineralógicos se llevaron a cabo en la Unidad Asociada al Centro de Astrobiología de la Universidad de Valladolid (Valladolid, España) mediante prototipos de instrumentos específicamente diseñados para misiones espaciales y otros equipos comerciales. Los espectros obtenidos se compararon con los de nuestra propia base de datos para cada una de las técnicas.

\section{Espectroscopia Raman}

La fuente de excitación para el análisis microRaman fue un láser Research Electro-Optics (REO) $(632.8 \mathrm{~nm})$. El espectrómetro usado fue un Holospec f/1.8i de Kaiser Optical Systems (KOSI), que trabaja en el rango 0 a $3800 \mathrm{~cm}^{-1}$, con una resolución espectral de $5 \mathrm{~cm}^{-1}$. Se utilizó una CCD DV420A-OE-130 de Andor y un cabezal Raman KOSI MKII, HFPHFC-S632.8 de Kaiser acoplado a una fibra óptica. El diámetro de los puntos analizados fue de $30 \mu \mathrm{m}$ y se realizaron mediante un microscopio Nikon Eclipse E600. La potencia del láser sobre la muestra fue de $10 \mathrm{~mW}$ a 50X. El tiempo de adquisición fue de 10 segundos y se hicieron 10 acumulaciones para cada espectro.

Para las determinaciones mineralógicas a partir de los espectros Raman obtenidos se utilizó un conjunto de algoritmos y la base de datos Raman que está siendo desarrollada en el ámbito de la misión planetaria ExoMars de la Agencia Espacial Europea (Hermosilla et al., 2012).

\section{Espectroscopia de infrarrojo}

Los espectros de infrarrojos con transformación de Fourier (FTIR) se obtuvieron con equipo Perkin Elmer Spectrum 100 FT-IR en modo de reflectancia acoplado a un accesorio universal ATR. Las muestras fueron escaneadas en el rango entre $550-4000 \mathrm{~cm}^{-1}$ con 10 acumulaciones. La resolución espectral fue de $4 \mathrm{~cm}^{-1}$. Los espectros de infrarrojo fueron comparados con los de nuestra propia base de datos mineralógica. 


\section{Difracción de Rayos X - Fluorescencia de Rayos $X$}

Los análisis combinados por XRD y XRF se realizaron con un equipo Terra basado en la tecnología desarrollada por la NASA para el instrumento CheMin a bordo del rover Curiosity de la misión MSL (Bish et al., 2013; Blake et al., 2013), comercializado por inXitu, Inc. (California). Las muestras fueron molidas previamente al análisis e introducidas en una célula vibratoria (Sarrazin et al., 2005). La radiación de rayos $\mathrm{X}$ procede de una fuente de $\mathrm{Co}$ $(10 \mathrm{~W})$. El equipo utiliza una CCD desarrollada por Andor y la muestra fue escaneada en el rango 5-55 del ángulo 20. El rango de FRX medido estuvo entre 2.5 y $10 \mathrm{keV}$. La resolución de los difractogramas es de $0.3^{\circ}$ del ángulo $2 \theta$, mientras que para los espectros de XRF fue de 250-300 eV. Más especificaciones sobre este instrumento se pueden encontrar en Bish et al. (2013) y Blake et al. (2013). Para el tratamiento de los espectros de difracción de rayos-X se dispuso del programa $\mathrm{X}$-Powder, basado en los algoritmos y la base de datos desarrollada para la misión MSL de la NASA (Martín, 2004).

\section{LIBS (Laser Induced Breakdown Spectroscopy)}

Para los análisis LIBS se utilizó un láser de doble frecuencia BIG SKY Ultra CFR Nd:YAG ( $1 \mathrm{~Hz}, 45 \mathrm{~mJ}$ at $532 \mathrm{~nm}, 6 \mathrm{~ns}$ de anchura de pulso) que se focalizó usando una lente convergente de $70 \mathrm{~mm}$ diámetro, y que permitió analizar puntos de $150 \mu \mathrm{m}$ sobre la superficie de la muestra sin necesidad de pre tratamiento. Los análisis se realizaron en condiciones atmosféricas terrestres. Las emisiones ópticas de la pluma de plasma fueron recogidas con un ángulo de $30^{\circ}$ mediante un colector ME-OPT-0007 de Andor. El espectrómetro utilizado fue un Mechelle 5000 de Andor, con una resolución mejor de $0.5 \pm 0.05 \mathrm{~nm}$ y que operó en el rango $200-975 \mathrm{~nm}$. El espectrómetro está acoplado a una cámara ISTAR 334T de Andor. El retraso entre el pulso del láser y la adquisición de la emisión fue de $1 \mu \mathrm{s}$, mientras que el tiempo de adquisición fue de $8 \mu \mathrm{s}$. Cada análisis representa la media de 10 pulsos del láser. Los espectros LIBS fueron comparados con los de nuestra propia base de datos mineralógica, que comprende más de 60 compuestos.
Elementos como azufre y el oxígeno, y otros como carbono, cloro, nitrógeno y flúor son problemáticos para ser detectados en condiciones de atmósfera terrestre. Esto se debe a que dichos elementos se recombinan fácilmente entre sí y con el oxígeno atmosférico para dar lugar rápidamente a gases diatómicos $\left(\mathrm{Cl}_{2}, \mathrm{~F}_{2}, \mathrm{O}_{2}\right)$ y óxidos gaseosos $\left(\mathrm{SO}_{2}, \mathrm{CO}_{2}\right.$, $\mathrm{NO}_{2}$ ) que escapan rápidamente de la pluma de plasma generada por el láser, por lo que su identificación no es posible. En los casos concretos del nitrógeno y el oxígeno, aunque estos elementos si son detectables mediante LIBS, su gran abundancia en el aire hace que su determinación en muestras en condiciones atmosféricas no sea posible. Estos inconvenientes se pueden evitar trabajando bajo condiciones de alto vacío (Effenberger \& Scott, 2010).

Teniendo en cuenta que la superficie de Marte presenta una atmósfera muy tenue y una baja concentración de gases (en particular oxígeno), la recombinación de los elementos citados anteriormente con oxígeno se minimiza prácticamente en su totalidad, permitiendo la identificación de estos átomos.

\section{Resultados y discusión}

\section{Yeso}

Tanto las espectroscopias Raman (Fig. 2) e IR (Fig. 3) como la DRX (Fig. 4) confirman la naturaleza yesífera de los grandes cristales de la geoda de Pulpí (Fig. 1A). Los espectros Raman y de IR obtenidos no muestran fluorescencia, presentan bandas perfectamente definidas y una relación señal/ruido extremadamente alta, del orden de $3 \cdot 10^{4}$ a 1 para la banda Raman principal situada a $1008 \mathrm{~cm}^{-1}$, correspondiente al modo de vibración $v_{1}$ del grupo $\mathrm{SO}_{4}$.

Desde el punto de vista geoquímico, los análisis cualitativos mediante FRX detectaron Ca como el elemento mayoritario, así como trazas de $\mathrm{Cr}$ y $\mathrm{Fe}$ (Fig. 5). Los espectros de FRX obtenidos mediante el instrumento Terra, gemelo del CheMin a bordo del vehículo Curiosity, presentan dos señales características a $6.93 \mathrm{keV}$ y $7.65 \mathrm{keV}$ que son debidas al Co de la fuente de rayos-X del equipo (Fig. 5). Por su lado, la espectroscopia LIBS detectó exclusivamente $\mathrm{Ca}$ en el yeso de Pulpí (Fig. 6).

Tanto la FRX como el LIBS no permitieron detectar $\mathrm{S}$ y $\mathrm{O}$, dos de los elementos mayoritarios en el 


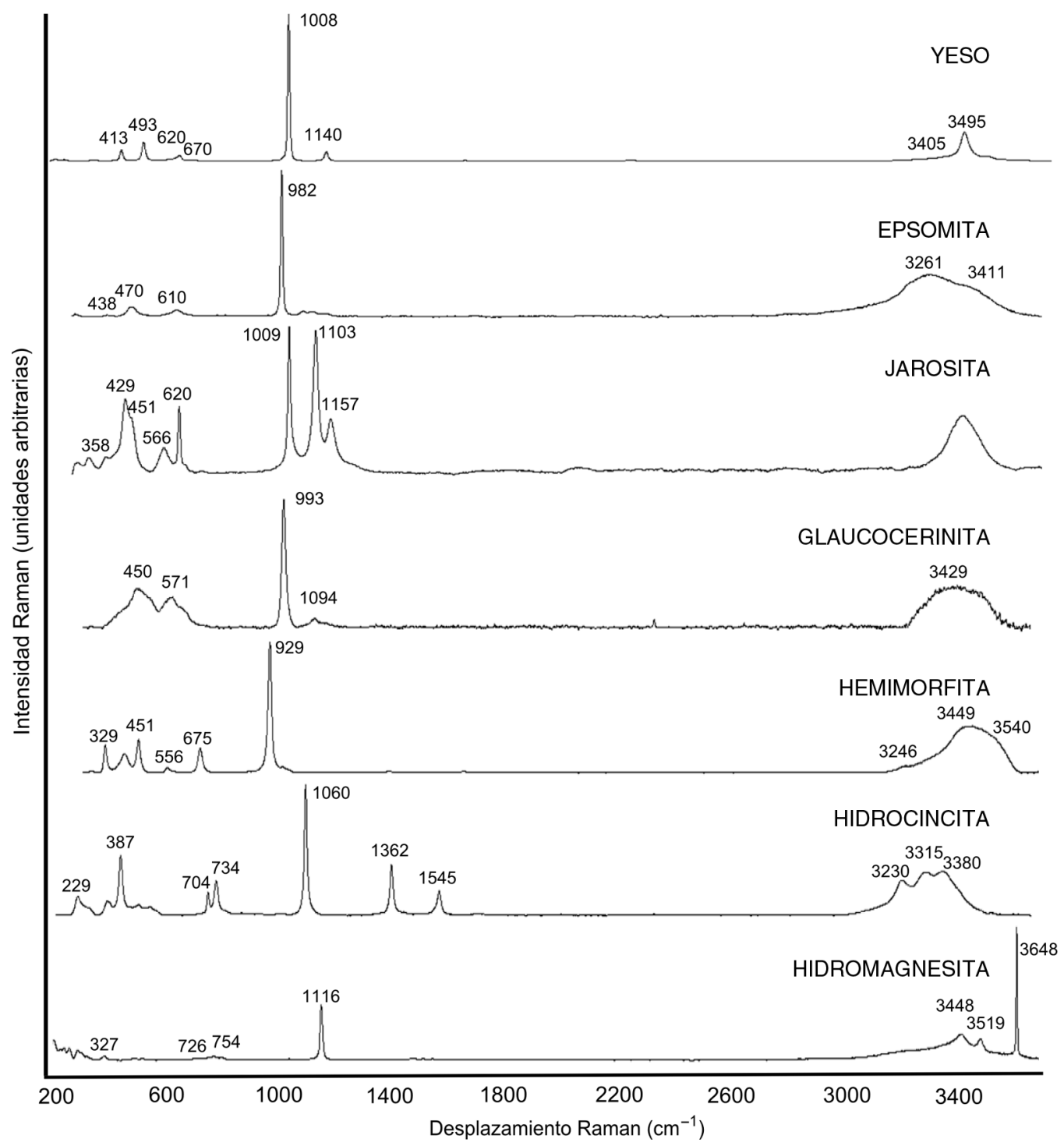

Fig. 2.-Espectros Raman de los minerales hidratados estudiados.

yeso. En el caso de la FRX, se debe a que la energías $\mathrm{K}_{\alpha}(0,52 \mathrm{keV})$ del oxígeno y las $\mathrm{K}_{\alpha}(2,31 \mathrm{keV})$ y $\mathrm{K}_{\beta}(2,46 \mathrm{keV})$ del azufre se encuentran por debajo de $2.5 \mathrm{keV}$, el límite inferior del rango de detección del instrumento. Del mismo modo, elementos con energías de ionización superiores a $10 \mathrm{keV}$ (Z superior a 32 , Germanio; $\mathrm{K}_{\alpha}=9,89 \mathrm{keV}$ ) no son detectables mediante esta técnica, lo que incluye elementos comunes como pueden ser el $\mathrm{Ba}, \mathrm{As}, \mathrm{Pb}$ o $\mathrm{Sr}$.

En cuanto al origen de los depósitos de yeso de la Mina Rica, García-Guinea et al. (2002) propusieron un modelo que se podría resumir en dos etapas: (1) formación de oquedades en la roca de caja carbonática como resultado de la reacción entre fluidos hidrotermales y las dolomías, e (2) infiltración de aguas ricas en sulfatos como resultado de la oxidación de depósitos de sulfuros presentes en la mina, así como contribuciones de fluidos de procedencia marina que dieron lugar a la precipitación de grandes cristales de yeso a temperatura más baja.

En cuanto a los yesos marcianos descubiertos hasta la fecha, la mayoría se ubican en el Olympia Planum, principalmente en forma de arenas yesíferas que constituyen densos campos de dunas en torno al 


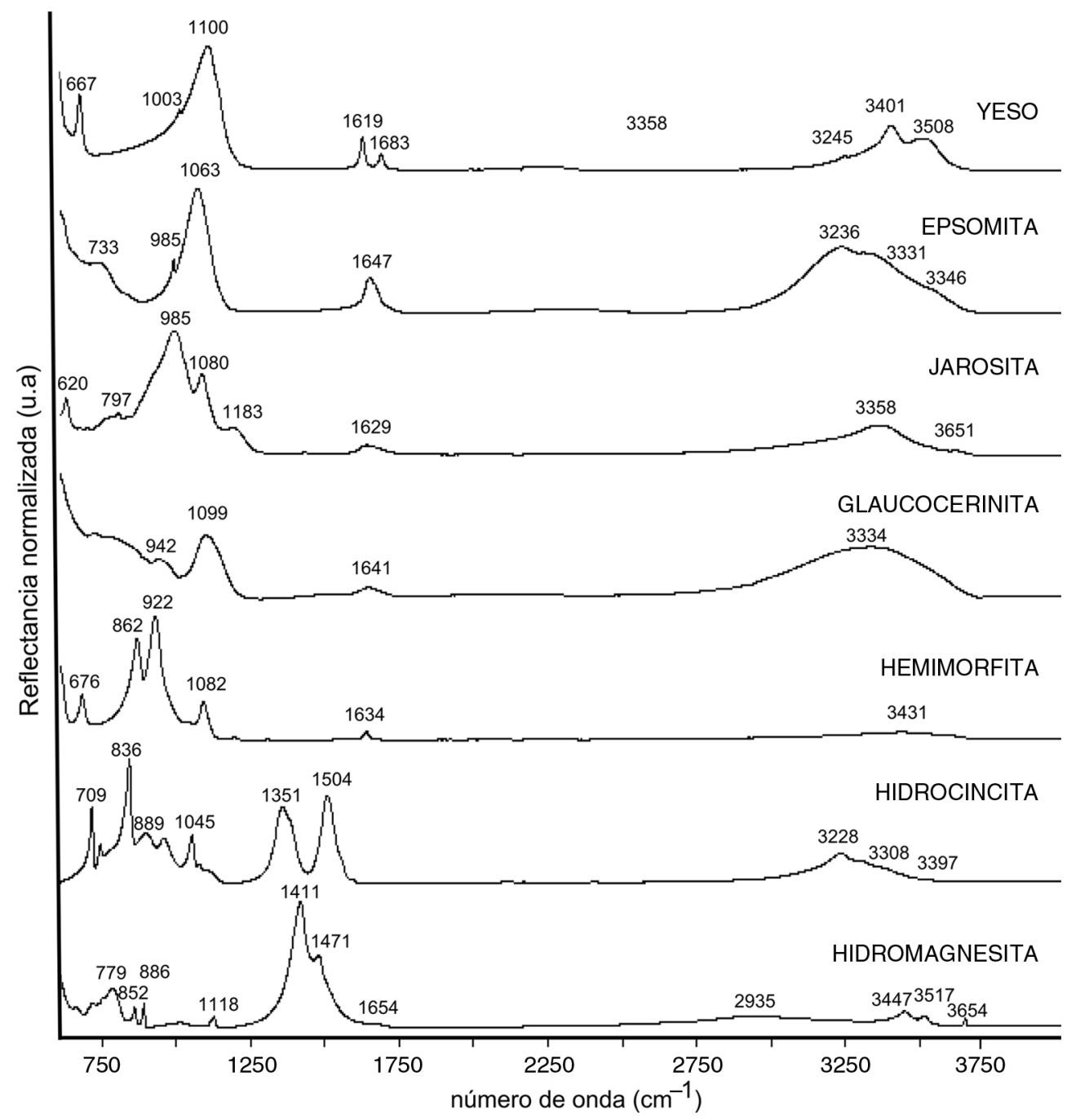

Fig. 3.-Espectros de infrarrojo de los minerales hidratados estudiados.

Polo Norte marciano (Massé et al., 2012). El origen de estos yesos está relacionado con la alteración del regolito basáltico, rico en minerales sulfurosos, debido a ablación regresiva producida por el hielo en el escarpe que bordea el casquete polar y la acción de los vientos (Massé et al., 2012).

Los mecanismos implicados en la precipitación de estos depósitos de yeso podrían diferir considerablemente de los descritos para el yeso de la Mina Rica de Pulpí, relacionado con procesos hidrotermales. Sin embargo, estos yesos no son los únicos descritos en Marte hasta la fecha. De hecho, en 2011 el rover Opportunity encontró venas minerales de color claro, constituidas por yeso, que podría haberse generado a partir de fluidos hidrotermales (Showstack, 2011), en un proceso similar al que tuvo lugar en la Mina Rica de Pulpí.

\section{Sulfatos hidratados de magnesio}

En los depósitos blanquecinos y pulverulentos que aparecen sobre algunas galerías mineras de la Mina Rica (Fig. 1B), se ha detectado epsomita y hexahidrita mediante espectroscopia Raman (Fig. 2). Por su lado, la espectroscopia de IR confirmó la presencia de sulfatos de $\mathrm{Mg}$ hidratados (Fig. 3), aunque esta técnica no es capaz de detectar pequeñas diferencias en el grado de hidratación de minerales de forma tan precisa como la espectroscopia Raman. La DRX confirmó la presencia de epsomita y hexahidrita, y también 


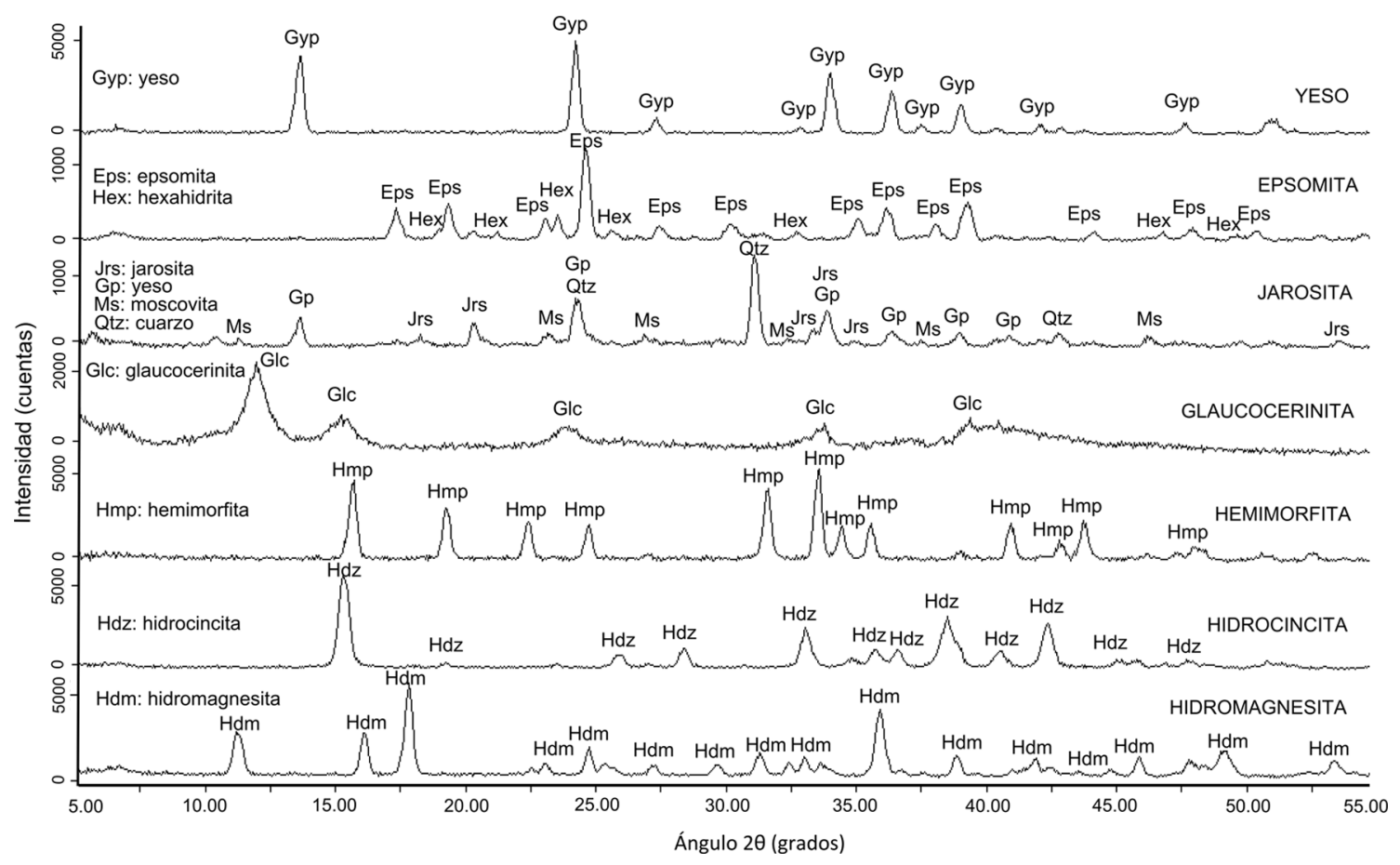

Fig. 4.-Difracción de rayos $\mathrm{X}$ de los minerales hidratados estudiados.

identificó wattevilleita $\left(\mathrm{Na}_{2} \mathrm{Ca}\left(\mathrm{SO}_{4}\right)_{2} \cdot 4 \mathrm{H}_{2} \mathrm{O}\right)$ (Fig. 4). La composición elemental de la muestra fue corroborada mediante FRX, que detectó $\mathrm{Ca}$, además de trazas de Fe (Fig. 5), mientras que el $\mathrm{Mg}$ no fue identificado debido a las limitaciones del instrumento citadas anteriormente. De forma similar, el LIBS identificó $\mathrm{Mg}$, además de $\mathrm{Ca}$, así como trazas de $\mathrm{Al}$ (Fig. 6).

La precipitación de sulfatos de $\mathrm{Mg}$ en forma de eflorescencias y costras en la Mina Rica está ligada al proceso de evaporación de agua capilar que fluye entre planos y fracturas de las filitas y dolomías de la roca de caja. La humectación de estos materiales se produce debido a la infiltración de agua de lluvia procedente de la superficie y a los procesos de condensación que disuelven y removilizan sulfatos heredados de las fases freáticas anteriores, así como elementos metálicos, principalmente calcio y magnesio de las dolomías. Los procesos de evaporación dan lugar a la sobresaturación de la solución y a la precipitación de sulfatos de $\mathrm{Mg}$ con distintos grados de hidratación.

La detección de sulfatos de $\mathrm{Mg}$ hidratados en Marte se reduce hasta la fecha al hallazgo de kieserita $\left(\mathrm{MgSO}_{4} \cdot \mathrm{H}_{2} \mathrm{O}\right)$ (Gendrinetal., 2005; Wrayetal., 2010). Sin embargo, otros sulfatos de $\mathrm{Mg}$ polihidratados tales como la starkeyita $\left(\mathrm{MgSO}_{4} \cdot 4 \mathrm{H}_{2} \mathrm{O}\right)$, pentahidrita $\left(\mathrm{MgSO}_{4} \cdot 5 \mathrm{H}_{2} \mathrm{O}\right)$, hexahidrita $\left(\mathrm{MgSO}_{4} \cdot 6 \mathrm{H}_{2} \mathrm{O}\right)$, epsomita $\left(\mathrm{MgSO}_{4} \cdot 7 \mathrm{H}_{2} \mathrm{O}\right)$ y meridianita $\left(\mathrm{MgSO}_{4} \cdot 11 \mathrm{H}_{2} \mathrm{O}\right)$ podría estar presentes. En el caso de la kieserita, ésta ha sido detectada en asociación con minerales arcillosos en el Cráter Gale, en el área de aterrizaje de la misión MSL de la NASA (Wiseman et al., 2008; Wray et al., 2010). La meteorización del regolito marciano en condiciones acuosas pudo producir la aparición de arcillas. Posteriormente, procesos de intensa evaporación de una solución rica en magnesio y sulfatos heredados de etapas hidrotermales previas podría haber dado lugar a sulfatos de $\mathrm{Mg}$ hidratados, similares a los observados en la Mina Rica de Pulpí.

\section{Jarosita}

En las muestras de materiales amarillentos que aparecen en forma de venas en el interior de la roca de caja de la Mina Rica se ha detectado jarosita, cuarzo, yeso y pequeñas cantidades de moscovita mediante DRX (Fig. 4). Esta técnica no permite distinguir entre diferentes minerales miembros de grupo de la jarosita, con fórmula general $\left(\mathrm{MFe}_{3}\left(\mathrm{SO}_{4}\right)_{2}(\mathrm{OH})_{6}\right)$, 

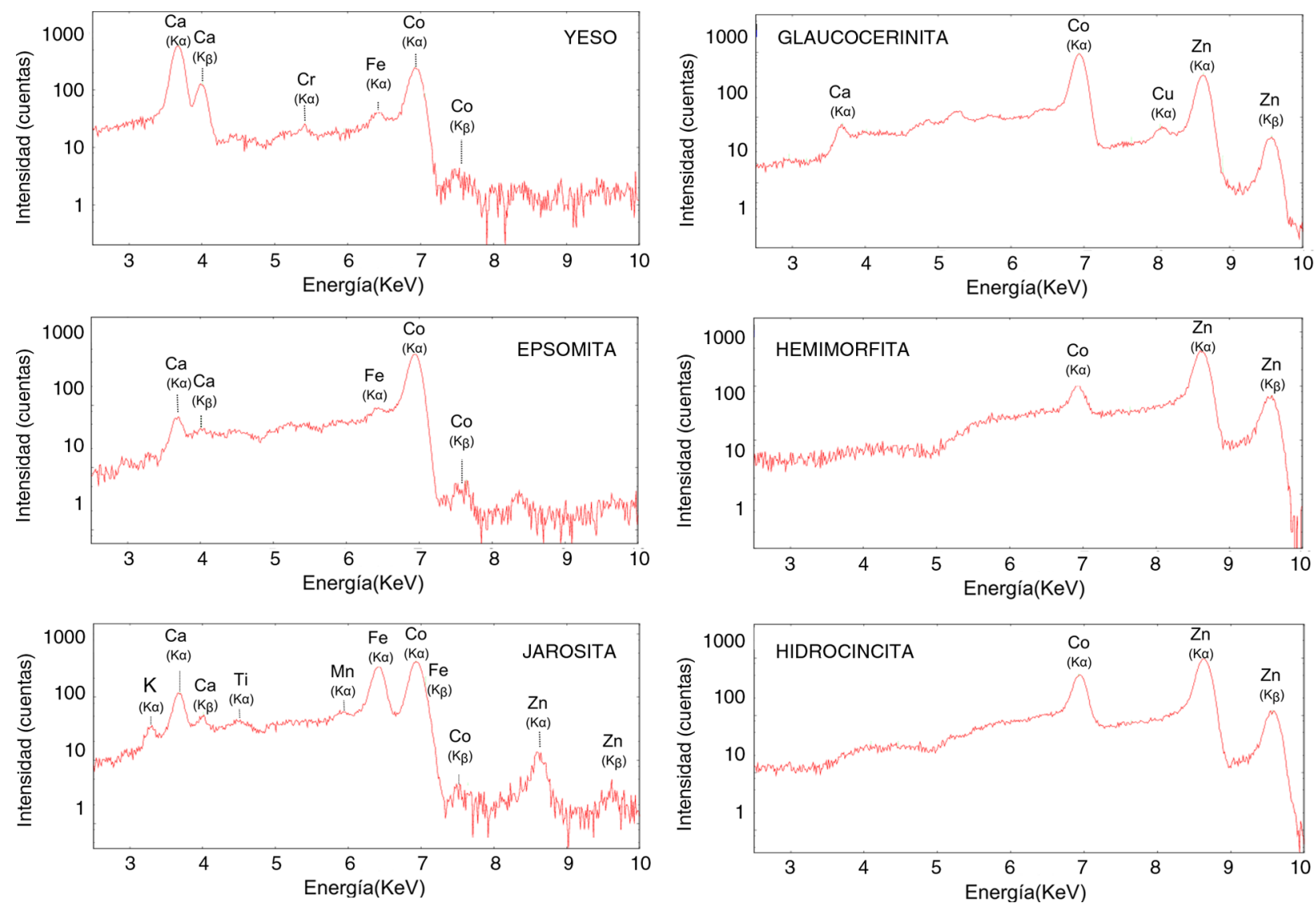

Fig. 5.-Fluorescencia de rayos $X$ de los minerales hidratados estudiados. Las señales de Co se deben a la fuente de rayos $X$ y no a que este elemento esté presente en las muestras. La muestra de hidromagnesita no produjo señales de fluorescencia en el rango de elementos estudiado.

donde $\mathrm{M}$ puede ser $\mathrm{K}^{+}, \mathrm{Ag}^{+}, \mathrm{NH}_{4}^{+}, \mathrm{Na}^{+}, \mathrm{Pb}^{2+}$ o $\mathrm{H}_{3} \mathrm{O}^{+}$. Por el contrario, la espectroscopia Raman es una técnica eficaz para discernir entre estos minerales (Frost et al., 2006). Mediante espectroscopia Raman se ha detectado jarosita $\left(\mathrm{KFe}_{3}\left(\mathrm{SO}_{4}\right)_{2}(\mathrm{OH})_{6}\right)$ y natrojarosita $\left(\mathrm{NaFe}_{3}\left(\mathrm{SO}_{4}\right)_{2}(\mathrm{OH})_{6}\right)$, en base a pequeñas diferencias en los perfiles Raman (Frost et al., 2006).

En cuanto a la composición química de esta jarosita, la FRX encontró $\mathrm{K}$ y Fe, además de Ca y Zn, así como trazas de Ti y Mn (Fig. 5). Esta técnica no detectó $\mathrm{Na}$ ya que su energía de emisión $\left(\mathrm{K}_{\alpha}=1,04 \mathrm{keV}\right)$ está por debajo del rango de detección del instrumento $(2,5 \mathrm{keV})$. Por su lado, el LIBS identificó $\mathrm{Fe}, \mathrm{K}$, y Na, confirmando la naturaleza mineralógica de la muestra, además de $\mathrm{Zn}, \mathrm{Al}$, y Si (Fig. 6). La detección de estos dos últimos elementos está en consonancia con la presencia de cuarzo y moscovita, mientras que el Zn podría encontrarse en solución sólida con los elementos metálicos mayoritarios de la jarosita, en particular reemplazando al $\mathrm{Fe}$, con similar radio iónico y carga.

La génesis de estos depósitos de jarosita parece estar ligada a procesos de alteración hidrotermal de materiales metamórficos en condiciones ácidas y de alta temperatura. De hecho, la presencia de trazas de moscovita en esta muestra corrobora esta hipótesis. Cabe destacar que esta mina se encuentra a tan solo $20 \mathrm{~km}$ de distancia del Barranco del Jaroso (Martínez-Frías, 1998), considerado como la localización tipo a escala mundial de la jarosita, por lo que los eventos hidrotermales que dieron lugar a la precipitación de este mineral pudieron ser similares.

La jarosita en Marte ha sido identificada por el espectrómetro Mössbauer del rover Opportunity en 


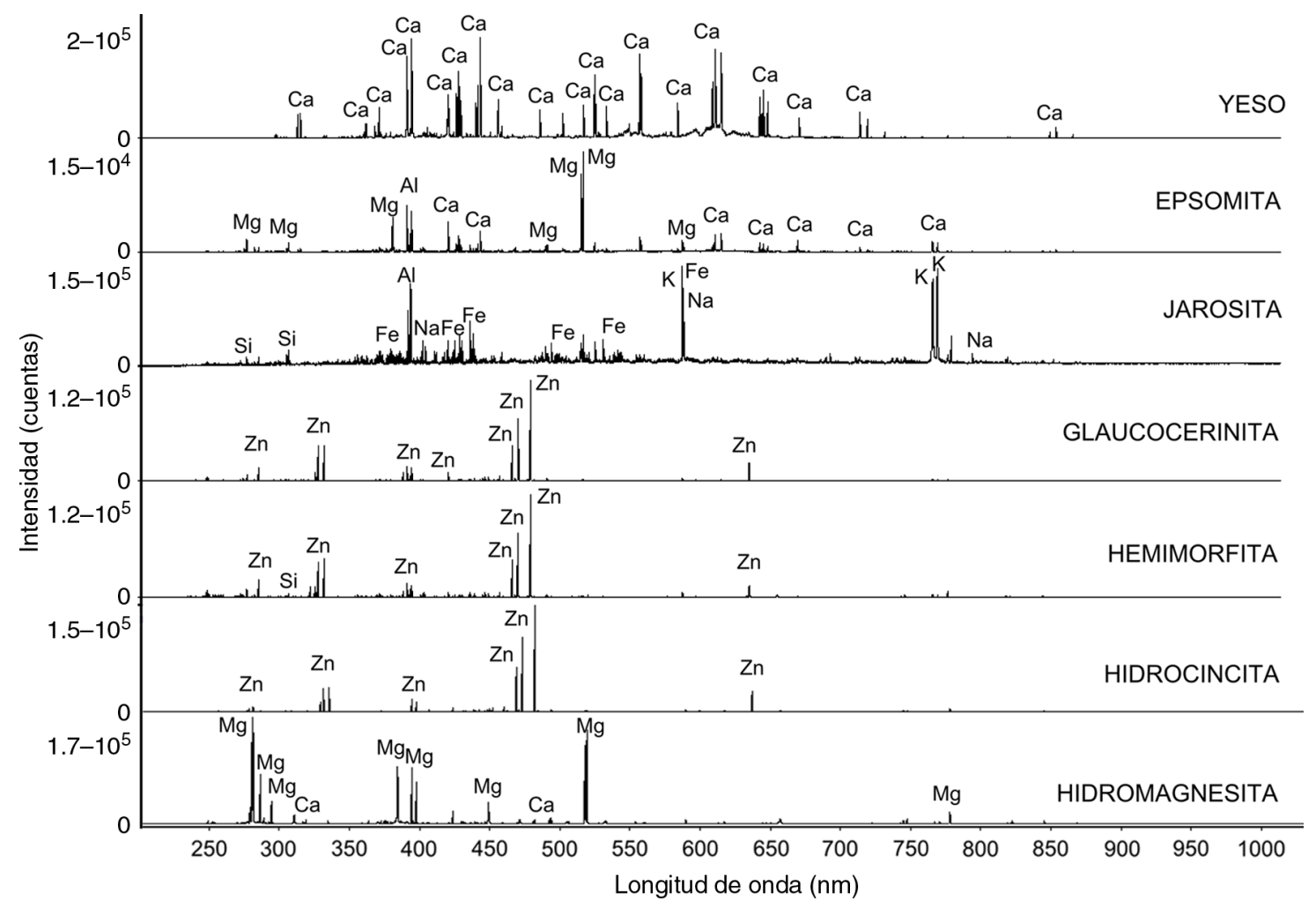

Fig. 6.-Espectroscopia LIBS de los minerales hidratados estudiados.

el Meridiani Planum, y su presencia ha sido considerada como una evidencia inequívoca de que condiciones húmedas y ácidas ocurrieron durante algunas etapas geológicas primitivas del Planeta Rojo (Klingelhöfer et al., 2004; Squyres et al., 2004). La alteración hidrotermal de materiales primitivos podría haber dado lugar a la aparición de jarosita en Marte, en un proceso similar al que generó las mineralizaciones encontradas en la Mina Rica de Pulpí.

\section{Glaucocerinita}

El análisis mineralógico de los microgours blancos y azules formados en la mina Su Zurfuru han revelado que están compuestos principalmente por glaucocerinita $\left((\mathrm{Zn}, \mathrm{Cu})_{5} \mathrm{AI}_{3}(\mathrm{OH})_{16}\left(\mathrm{SO}_{4}\right)_{1.5} \cdot 9 \mathrm{H}_{2} \mathrm{O}\right)$, detectada mediante espectroscopias Raman y de IR (Figs. 2 y 3), así como por DRX (Fig. 4). Además en el caso de los gours blancos se han detectado yeso y trazas de fluorita (Gázquez et al., 2013a). La FRX reveló la presencia de $\mathrm{Zn}$ como elemento mayoritario y concentraciones menores de Ca (Fig. 5), lo que corrobora los resultados mineralógicos obtenidos. La espectroscopia LIBS detectó exclusivamente Zn en estas muestras (Fig. 6). Microanálisis previos mediante energía dispersiva de rayos- $\mathrm{X}$ también detectaron $\mathrm{Cu}, \mathrm{S}, \mathrm{O}$ y Al en las proporciones propias a este mineral (Gázquez et al., 2013a).

$\mathrm{El}$ origen de estas formaciones en la mina $\mathrm{Su}$ Zurfuru está relacionado con la oxidación de mineralizaciones presentes en la roca de caja, principalmente sulfuros de $\mathrm{Zn}$ y $\mathrm{Cu}$ (esfalerita y calcopirita). El agua rica en sulfuros disueltos emerge en las galerías, produciéndose así la oxidación de los sulfuros a sulfatos en un medio oxigenado. La hidrólisis de minerales de arcillas que constituyen el sustrato sobre el que aparece la glaucocerinita aporta el aluminio necesario para la precipitación de este mineral.

Aunque la presencia de sulfatos hidratados de $\mathrm{Zn}$ y/o $\mathrm{Cu}$ no ha sido constatada en la superficie marciana hasta la fecha, el rover Opportunity encontró rocas con alto contenido en $\mathrm{Zn}$ en el Cráter Endeavor. 
Este hecho sumado a la presencia de otros sulfatos detectados en diversos puntos del planeta, sugieren que este tipo de minerales cuya génesis está íntimamente ligada a la presencia de agua líquida, también podría ser detectado en Marte.

\section{Hemimorfita e Hidrocincita}

Los análisis mineralógicos de la gran colada de la Crovassa Quarziti mediante las tres técnicas empleadas han revelado la presencia de hemimorfita $\left(\mathrm{Zn}_{4} \mathrm{Si}_{2} \mathrm{O}_{7}(\mathrm{OH})_{2} \cdot \mathrm{H}_{2} \mathrm{O}\right)$ (Figs. 2,3 y 4 ). Por otro lado, la FRX (Fig. 5) al igual que el LIBS (Fig. 6) indican la presencia de importantes cantidades de Zn, corroborando los datos mineralógicos.

El origen de esta colada de grandes dimensiones pudo estar relacionado con la alteración del sustrato de cuarcita, debido a un flujo laminar de agua con $\mathrm{pH}$ alcalino en condiciones aéreas. Este mecanismo de alteración de materiales silíceos en medio básico ha sido observado también en otras cuevas del entorno de Crovassa Quarziti (Sauro et al., 2014). De este modo se produjo la alteración de la cuarcita y la precipitación de láminas de hemimorfita, que en algunas ocasiones se alternó con períodos de precipitación de óxidos de Fe y Mn, probablemente como consecuencia de pequeños cambios en el $\mathrm{pH}$ y la saturación en hemimorfita de la disolución.

En cuanto a los depósitos blancos en forma de láminas encontrados en la Cueva Massa Riccardo de la Mina San Giovanni, las técnicas mineralógicas empleadas coinciden en que se trata de hidrocincita $\left(\mathrm{Zn}_{5}\left(\mathrm{CO}_{3}\right)_{2}(\mathrm{OH})_{6}\right)$ (Figs. 2, 3 y 4). Tanto la FRX (Fig. 5) como el LIBS (Fig. 6) han detectado Zn en estos depósitos, por lo que confirman su naturaleza mineralógica.

La hidrocincita en esta cueva aparece sobre depósitos de óxidos de Fe y Mn y cubierta por capas de calcita espeleotémica lo que sugiere que estas mineralizaciones precipitaron en condiciones de transición entre una fase hidrotermal oxigénica de precipitación de óxidos y otra, probablemente vadosa o epifreática en la que se formaron láminas de calcita espeleotémica a modo de coladas. La hidrocincita podría marcar el cambio de condiciones relativamente ácidas a otras con un $\mathrm{pH}$ más elevado que posteriormente permitieron la precipitación de carbonato de calcio.
En el ámbito de la exploración marciana, la hemimorfita y la hidrocincita no han sido aún identificadas sobre la superficie del Planeta Rojo. No obstante, como se ha comentado anteriormente, el rover Opportunity de la NASA ha detectado recientemente rocas con alto contenido en Zn en el Cráter Endeavor. Este hecho permite especular sobre la posible presencia de sulfatos, silicatos y carbonatos de $\mathrm{Zn}$ en Marte, que podrían ser identificados en las próximas misiones de exploración marciana.

\section{Hidromagnesita}

Los depósitos de moonmilk de la cueva de El Soplao están constituidos esencialmente por hidromagnesita $\left(\mathrm{Mg}_{5}\left(\mathrm{CO}_{3}\right)_{4}(\mathrm{OH})_{2} \cdot 4 \mathrm{H}_{2} \mathrm{O}\right)$ que ha sido identificada mediante espectroscopias Raman (Fig. 2) y de IR (Fig. 3), así como DRX (Fig. 4) (Gázquez, 2012). En este caso, la FRX no ha sido capaz de detectar ningún elemento, ya que tanto el C, como el $\mathrm{Mg}$ y el $\mathrm{O}$, se encuentran fuera del rango de detección del instrumento. En cambio, la espectroscopia LIBS detectó $\mathrm{Mg}$ y pequeñas concentraciones de $\mathrm{Ca}$ (Fig. 6).

El moonmilk de hidromagnesita de la Cueva de El Soplao aparece en la Galería de El Bosque, encontrándose solamente sobre uno de los flancos de los espeleotemas. Este hecho apunta a que la dirección del aire juega un papel fundamental durante su desarrollo y sugiere que la precipitación directa a partir de una disolución rica en Mg es la explicación plausible para la génesis de estos depósitos (Gázquez et al., 2010; 2012b).

Calvin et al., (1994) relacionaron bandas de absorción en el infrarrojo tomadas por las sondas Mariner 6 y 7 con la presencia de carbonatos de magnesio hidratados, posiblemente hidromagnesita o artinita $\left(\mathrm{Mg}_{2} \mathrm{CO}_{3}(\mathrm{OH})_{2} \cdot 3 \mathrm{H}_{2} \mathrm{O}\right)$, en la región de Sinus Meridiani de Marte, mientras que también se ha sugerido su posible ocurrencia en la región de Terra Sabaea (Russell et al., 1999). Estos autores relacionan los depósitos de carbonatos magnésicos hidratados en Marte con la alteración de basaltos con alto contenido en $\mathrm{Mg}$ y komatitas en un ambiente acuoso y con alto contenido en $\mathrm{CO}_{2}$. La precipitación de hidromagnesita requiere de una elevada tasa de evaporación y procesos intensos de desgasificación que dan lugar a un $\mathrm{pH}$ de la 
disolución altamente alcalino. En muchos casos, la aparición de este tipo de espeleotemas magnésicos está relacionada con la actividad de microorganismos, cuyo metabolismo controla la concentración de especies de carbono disueltas en la disolución (Gázquez et al., 2012b). Por lo tanto, el análisis de depósitos de moonmilk en cavidades se postula como un campo de estudio de gran importancia en cuanto a la precipitación de carbonatos magnésicos hidratados en Marte y su posible relación con la presencia de microorganismos.

\section{Conclusiones}

Sulfatos, silicatos y carbonatos hidratados procedentes de ambientes subterráneos han sido identificados mediante técnicas mineralógicas y geoquímicas implicadas en misiones de exploración planetaria. Los resultados obtenidos en este estudio revelan que las técnicas espectroscópicas utilizadas (Raman e IR) son tan eficaces como las técnicas mineralógicas tradicionales (DRX) para la identificación de fases minerales, y en particular de fases hidratadas, que han sido propuestas como una de las mayores evidencias de la presencia de agua líquida durante algunas fases de la historia geológica del Planeta Rojo.

La espectroscopia Raman ha permitido diferenciar entre varios sulfatos de $\mathrm{Mg}$ (epsomita y hexahidrita) y dos minerales integrantes del grupo de la jarosita (jarosita y natrojarosita). Por otro lado, la DRX ha detectado varios sulfatos de Mg (epsomita, hexahidrita y wattevilleita) pero no ha permitido distinguir entre minerales distintos del grupo de la jarosita. En el caso de la espectroscopia de IR, su capacidad para detectar pequeñas diferencias entre minerales es más limitada aunque ha permitido identificar jarosita, sulfatos de Mg hidratados y la mayoría de minerales identificados mediante el resto de técnicas.

Las tres técnicas mineralógicas empleadas han permitido identificar con claridad sulfatos de $\mathrm{Ca}$, Fe y Zn hidratados (yeso, minerales del grupo de la jarosita y glaucocerinita), así como silicatos hidratados de Zn (hemimorfita) y carbonatos hidratados (hidrocincita e hidromagnesita). Las técnicas geoquímicas utilizadas (FRX y LIBS) han confirmado los datos mineralógicos obtenidos.
Cabe destacar que las espectroscopias Raman y de IR son técnicas capaces de identificar compuestos orgánicos en pequeñas proporciones en el interior de matrices inorgánicas, por lo que hay ciertas expectativas generadas para la detección de vestigios de vida en el marco de la misión ExoMars. Por otra parte, del conjunto de técnicas empleadas, la combinación Raman-LIBS se perfila como la opción más eficiente para la detección de minerales hidratados en condiciones marcianas, tanto por sus ventajas técnicas, como es el hecho de no requerir de preparación de muestra, como por sus capacidades analíticas y de identificación de compuestos y estructuras.

Algunos de los minerales estudiados en este trabajo han sido identificados también en la superficie de Marte, mientras que otros, como es el caso de los silicatos y sulfatos de Zn hidratados, podrían ser candidatos a estar presentes sobre la superficie marciana, como sugieren descubrimientos previos sobre la geoquímica de Marte. Los mecanismos de formación de minerales en condiciones acuosas observados en ambientes subterráneos, ya sean en condiciones hidrotermales o de baja temperatura, podrían ser análogos de los procesos mineralogenéticos acontecidos durante la historia geológica de Marte.

\section{AGRADECIMIENTOS}

Los autores agradecen la asistencia y ayuda prestada durante las campañas de muestreo a D. Manuel Guerrero, Angelo Naseddu, los Espeleoclubs Almería (Almería) y Domusnovas (Iglesias, Italia), así como a los gestores de la Cueva de El Soplao. Este trabajo ha sido financiado por los proyectos AYA2011-30291-C02-02 y AYA2012-39884-C02-02 (Ministerio de Ciencia e Innovación de España y Fondos FEDER de la UE). Los autores agradecen las correcciones y sugerencias hechas por el Dr. Rogelio Daniel Acevedo y un revisor anónimo.

\section{Referencias}

Arvidson, R.E.; Poulet, F.; Bibring, J.P.; Wolff, M.; Gendrin, A.; Morris, R.V.; Freeman, J.J.; Langevin, Y.; Mangold, N. \& Bellucci, G. (2005). Spectral reflectance and morphologic correlations in eastern Terra Meridiani, Mars. Science, 307: 1591-1594. http://dx.doi.org/10.1126/ science.1109509.

Baioni, D.; Zupan Hajna, N. \& Wezel, F.C. (2009). Karst landforms in a Martian evaporitic dome. Acta Carsologica, 38(1): 9-18.

Bibring, J.P.; Langevin, Y.; Gendrin, A.; Gondet, B.; Poulet, F.; Berthé, M.; Soufflot, A.; Arvidson, R.; Mangold, N.; 
Mustard, J.; Drossart, P. \& the OMEGA team. (2005). Mars surface diversity as revealed by the OMEGA/ Mars Express observations. Science, 307: 1576-1581. http://dx.doi.org/10.1126/science.1108806.

Bish, D.L.; Blake, D.F.; Vaniman, D.T; Chipera, S.J.; Morris, R.V.; Ming, D.W.; Treiman,A.H.; Sarrazin,P.; Morrison, S.M.; Downs, R.T.; Achilles, C.N.; Yen, A.S.; Bristow, T.F.; Crisp, J.A.; Morookian, J.M.; Farmer, J.D.; Rampe, E.B.; Stolper, E.M.; Spanovich, N.; MSL Science Team. (2013). X-ray Diffraction Results from Mars Science Laboratory: Mineralogy of Rocknest at Gale Crater. Science, 341: 1238932(1-5).

Bechstadt, T. \& Boni, M. (1996). Sedimentological, stratigraphical and ore deposits field guide of the autochtonous Cambro-Ordovician of Southwestern Sardinia. Memorie descrittive della Carta Geologica d'Italia, 48: 1-390.

Blake, D.F.; Morris, R.V.; Kocurek, G.; Morrison, S.M.; Downs, R.T.; Bish, D.; Ming, D.W.; Edgett, K.S; Rubin, D.; Goetz, W.; Madsen, M.B.; Sullivan, R.; Gellert, R.; Campbell, I.; Treiman, A.H.; McLennan, S.M.; Yen,A.S.; Grotzinger, J.; Vaniman, D.T.; Chipera, S.J.; Achilles, C.N.; Rampe, E.B; Sumner, D.; Meslin, P.Y.; Maurice, S.; Forni, O.; Gasnault, O.; Fisk, M.; Schmidt, M.; Mahaffy, P.; Leshin, L.A.; Glavin, D.; Steele, A.; Freissinet, C.; Navarro-González, R.; Yingst, R.A.; Kah, L.C.; Bridges, N.; Lewis, K.W.; Bristow, T.F.; Farmer, J.D.; Crisp, J.A.; Stolper, E.M.; Des Marais, D.J.; Sarrazin, P.; MSL Science Team. (2013). Curiosity at Gale Crater, Mars: Characterization and Analysis of the Rocknest Sand Shadow. Science, 341: http://dx.doi.org/10.1126/science. 1239505.

Boston, P.J.; Spilde, M.N.; Northup, D.E.; Melim, L.A.; Sorok, D.S.; Kleina, L.G.; Lavoie, K.H.;Hose, L.D.; Mallory, L.M.; Dahm, C.N.; Crossey, L.J. \& Schelble, R.T. (2001). Cave biosignature suites: microbes, minerals, and Mars. Astrobiology, 1: 25-54. http:// dx.doi.org/10.1089/153110701750137413.

Boston, P.J.; Frederick, R.D.; Welch, S.M.; Werker, J.; Meyer, T.R.; Sprungman, B.; Hildreth-Werker, V.; Thompson, S.L. \& Murphy, D.L. (2003). Human utilization of subsurface extraterrestrial environments. Gravitational and space Research. 16(2): 121-131.

Caddeo, G.A.; De Waele, J.; Frau, F. \& Railsback, L.B. (2011). Trace element and stable isotope data from a flowstone in a natural cave of the mining district of SW Sardinia (Italy): evidence for $\mathrm{Zn} 2+$ induced aragonite precipitation in comparatively wet climatic conditions. International Journal of Speleology. 40 (2): 181-190. http://dx.doi.org/10.5038/1827-806X.40.2.10.

Calaforra, J.M.; Moreno, R.; García-Guinea, J.; Guerrero, M. \& Romero, A., (2001). La geoda gigante de Pulpí (The giant Geode of Pulpí): Patrimonio geológico y minero. Medio Ambiente. 37: 42-43.

Calvin, W.M.; King, T.V.V. \& Clark, R. N. (1994). Hydrous carbonates on Mars?: Evidence from Mariner 6/7 infrared spectrometer and ground-based telescopic spectra. Journal of Geophysical Research: Planets. 99: E7, 14659-14675. http://dx.doi.org/ 10.1029/94JE01090.

Cushing, G.E. (2012). Candidate cave entrances on Mars. Journal of Caves and Karst Studies. 74(1): 33-47. http://dx.doi.org/10.4311/2010EX0167R.

De Waele, J.; Forti, P. \& Naseddu, A. (2013). Speleogenesis of a complex example of an exhumed sulphuric acid karst in Cambrian carbonates (Mount San Giovanni, Sardinia). Earth Surface Processes and Landforms, 38: 1369-1379.

Effenberger, A.J. Jr. \& Scott, J.R. (2010). Effect of atmospheric conditions on LIBS spectra. Sensors, 10: 4907-4925. http://dx.doi.org/10.3390/s100504907.

Fernández-Cortés, A.; Calaforra, J.M. \& García-Guinea, J. (2006). The Pulpí gigantic geode (Almería, Spain): geology, metal pollution, microclimatology and conservation. Environmental Geology, 50(5): 707-716. http://dx.doi.org/10.1007/s00254-006-0243-z.

Forti, P.; Galli, E.; Rossi, A. \& Naseddu, A. (2005). Studio mineralogico di una concrezione della Fontana dello Zinco (Miniera di Su Zurfuru, Fluminimaggiore). In: Le Grotte di Miniera: Tra economia mineraria ed economia turistica (De Waele J. \& Naseddu A., eds.). Memorie dell'Istituto Italiano di Speleologia, 17: 69-86.

Frost, R.L.; Will, R.A.; Weir, M.L.; Martens, W. \& Mills, S. (2006). A Raman spectroscopy study of selected natural jarosites. Spectrochimica Acta Part A, 63: 1-8. http://dx.doi.org/10.1016/j.saa.2005.03.034.

García-Guinea, J.; Morales, S.; Delgado, A.; Recio, C. \& Calaforra, J.M. (2002). Formation of gigantic gypsum crystals. Journal of the Geological Society of London, 159: 347-350. http://dx.doi.org/10.1144/0016-764902001 .

Gázquez, F.; Delgado-Huertas, A.; Forti, P.; Stöll, H. \& Calaforra, J.M. (2010). Variabilidad paleoambiental en la Cueva de El Soplao (Cantabria) a partir de la diversidad mineralógica de sus espeleotemas. In: Cuevas: Patrimonio, Naturaleza, Cultura y turismo. (J.J. Durán \& F.Carrasco, eds.). Madrid. Asociación de Cuevas turísticas. 293-304 pp.

Gázquez, F.; Calaforra, J.M. \& Forti, P. (2011). Black $\mathrm{Mn}-\mathrm{Fe}$ Crusts as Markers of Abrupt Palaeoenvironmental Changes in El Soplao Cave (Cantabria. Spain). International Journal of Speleology, 40: 163-169. http://dx.doi.org/10.5038/1827-806X.40.2.8.

Gázquez, F. (2012). Registros paleoambientales a partir de espeleotemas yesíferos y carbonáticos. Tesis Doctoral. Universidad de Almería, 381 pp.

Gázquez, F.; Calaforra, J.M.; Rull, F.; Forti, P. \& GarcíaCasco, A. (2012a). Organic matter of fossil origin in the amberine speleothems from El Soplao Cave (Cantabria. Northern Spain). International Journal of Speleology, 41: 113-123. http://dx.doi. org/10.5038/1827-806X.41.1.12. 
Gázquez, F.; Calaforra, J.M. \& Sanna, L. (2012b). Precipitación de moonmilk, un proceso natural en cavidades: revisión y nuevos casos de estudio. In: Las cuevas turísticas como activos económicos: conservación e innovación, Asociación Española de Cuevas Turísticas (J.J. Durán \&, P.A. Robledo, eds.), 47-60 pp.

Gázquez, F.; Rull, F.; Medina, J.; Calaforra, J.M.; Forti, P.; De Waele, J.; Venegas, G. \& Sanz, A. (2013a). Glaucocerinite forming gours in Su Zurfuru Mine (Sardinia, Italy). Revista de la Sociedad Española de Mineralogía, Macla, 17: 53-54.

Gázquez, F.; Calaforra, J.M.; Forti, P.; De Waele, J.; Sanna, L.; Rull, F. \& Sanz, A. (2013b). Corrosion of calcite crystals by metal-rich mud in caves: Study case in Crovassa Ricchi in Argento Cave (SW Sardinia, Italy). Geomorphology, 198: 133-146. http://dx.doi.org/ 10.1016/j.geomorph.2013.05.022.

Gázquez, F.; Calaforra, J.M.; Forti, P.; Stoll, H.; Ghaleb, B. \& Delgado-Huertas, A. (2014). Paleoflood events recorded by speleothems in caves. Earth Surface Process and Landforms (aceptado). http://dx.doi.org/ 10.1002/esp.3543.

Gendrin, A.; Mangold, N.; Bibring, J.P.; Langevin, Y; Gondet, B.; Poulet, F.; Bonello, G.; Quantin, C.; Mustard, J.; Arvidson, R. \& LeMouélic, S. (2005). Sulfates in Martian layered terrains: The OMEGA/ Mars Express view. Science, 307: 1587-1591. http:// dx.doi.org/10.1126/science.1109087.

Hermosilla, I.; Lopez-Reyes, G.; Catalá, A.; Sanz, A.; Llanos D.R. \& Rull, F. (2012). Raman spectra processing algorithms and database for RLS-ExoMars. EPSC Abstracts, 7 EPSC2012-567-1.

Kisdi, Á. \& Tatnall, A.R.L. (2011). Future robotic exploration using honeybee search strategy: Example search for caves on Mars. Acta Astronautica, 68: 1790-1799. http://dx.doi.org/10.1016/j.actaastro.2011.01.013.

Klingelhöfer, G.; Morris, R.V.; Bernhardt, B.; Schröder, C.; Rodionov, D.S.; de Souza, P.A. Jr.; Yen, A.; Gellert, R.; Evlanov, E.N.; Zubkov, B.; Foh, J.; Bonnes, U.; Kankeleit, E.; Gütlich, P.; Ming, D.W.; Renz, F.; Wdowiak, T.; Squyres, S.W. \& Arvidson, R.E. (2004). Jarosite and Hematite at Meridiani Planum from Opportunity's Mössbauer Spectrometer. Science, 306: 1740-1745. http://dx.doi.org/10.1126/science. 1104653.

Lane, M.D.; Darby Dyar, M. \& Bishop, J.L. (2004). Spectroscopic evidence for hydrous iron sulfate in the Martian soil. Geophysical Research Letters, 31, L19702, $1-4$.

Langevin, Y.; Poulet, F.; Bibring, J.P. \& Gondet, B. (2005). Sulfates in the north polar region of Mars detected by OMEGA/Mars Express. Science, 307; 1584-1586. http://dx.doi.org/10.1126/science.1109091.

Lanza, N.L., Wiens, R.C.; Clegg, S.M.; Ollila, A.M.; Humphries, S.D.; Newsom, H.E. \& Barefield, J.E. (2010). Calibrating the ChemCam laser-induced breakdown spectroscopy instrument for carbonate minerals on Mars. Applied Optic, 49: C211-C217. http://dx.doi.org/10.1364/AO.49.00C211.

Ling, Z.C. \& Wang, A. (2010). A systematic spectroscopic study of eight hydrous ferric sulfates relevant to Mars. Icarus, 209: 422-433. http://dx.doi. org/10.1016/j.icarus.2010.05.009.

López-Reyes, G.; Rull,F.; Venegas, G.;Westall, F.; Foucher, F.; Bost, N.; Sanz, A.; Catalá-Espí, A.; Vegas A.; Hermosilla, I.; Sansano, A. \& Medina, J. (2013). Analysis of the scientific capabilities of the ExoMars Raman Laser Spectrometer instrument. European Journal of Mineralogy, 25(5): 721-733. http://dx.doi. org/10.1127/0935-1221/2013/0025-2317.

Martín, D. (2004). Programa para el análisis por difracción de rayos X. Método en Polvo (XPowder). Universidad de Granada.

Martínez-Frías, J. (1998). An ancient Ba-Sb-Ag-Fe-Hg bearing hydrothermal system in SE Spain. Episodes, 21: 248-251.

Martínez-Frías, J.; Lunar, R.; Rodríguez-Losada, J.A. \& Delgado, A. (2004). The volcanism-related multistage hydrothermal system of El Jaroso (SE Spain): implications for the exploration of Mars. Earth Planets Space, 56: v-viii.

Martinez-Frías, J.; Amaral, G.; Vázquez, L. (2006). Astrobiological significance of minerals on Mars surface environment. Review in Environmental Sciences and Biotechnology, 5: 219-231. http://dx.doi.org/10.1007/ s11157-006-0008-x.

Massé, M.; Bourgeois, O.; Le Mouélic, S.; Verpoorter, C.; Spiga, A. \& Le Deit, L. (2012). Wide distribution and glacial origin of polar gypsum on Mars. Earth and Planetary Science Letters, 317-318: 44-55. http://dx. doi.org/10.1016/j.epsl.2011.11.035.

Milliken, R.E.; Mustard, J.F.; Poulet, F.; Jouglet, D.; Bibring, J.P.; Gondet, B. \& Langevin, Y. (2007). Hydration state of the martian surface as seen by Mars Express OMEGA. 2. H2O content of the surface. Journal of Geophysical Research, 112 (E8): E08S07. http://dx.doi.org/10.1029/2006JE002853.

Mustard, J.F.; Murchie, S.L.; Pelkey, S.M.; Ehlmann, B.L.; Milliken, R.E.; Grant, J.A.; Bibring, J-P.; Poulet, F.; Bishop, J.; Noe Dobrea, E.; Roach, L.; Seelos, F.; Arvidson, R.E.; Wiseman, S.; Green, R.; Hash, C.; Humm, D.; Malaret, E.; McGovern, J.A.; Seelos, K.; Clancy, T.; Clark, R.; Marais, D.; Izenberg, N.; Knudson, A.; Langevin, Y.; Martin, T.; McGuire, P.; Morris, R.; Robinson, M.; Roush, T.; Smith, M.; Swayze, G.; Taylor, H.; Titus, T. \& Wolff, M. (2008). Hydrated silicate minerals on Mars observed by the Mars Reconnaissance Orbiter CRISM instrument. Nature, 454: 305-309. http://dx.doi.org/10.1038/ nature07097.

Pagliara, A.; De Waele, J.; Forti, P.; Galli, E. \& Rossi, A. (2010). Speleothems and speleogenesis of the hypogenic Santa Barbara cave system (South-West Sardinia, Italy). Acta Carsologica, 39(3): 551-564. 
Quesada, S.; Robles, S. \& Rosales, I. (2005). Depositional architecture and transgressive-regressive cycles within Liassic backstepping carbonates ramps in the Basque-Cantabrian Basin, N Spain. Journal of the Geological Society of London, 162: 531-548. http:// dx.doi.org/10.1144/0016-764903-041.

Rull, F. \& Martinez-Frías, J. (2006). Raman spectroscopy goes to Mars. Spectroscopy Europe, 1: 18-21.

Russell, M.J.; Ingham, J.K.; Zedef, V.; Maktav, D.; Sunar, F.; Hall, A.J. \& Fallick, A.E. (1999). Search for signs of ancient life on Mars: expectations from hydromagnesite microbialities, Salda Lake, Turkey. Journal of the Geological Society of London, 156: 869-888. http://dx.doi.org/10.1144/gsjgs.156.5.0869.

Sarrazin, P.; Chipera, S.; Bish, D.; Blake, D. \& Vaniman, D. (2005). Vibrating sample holder for XRD analysis with minimal sample preparation. International Centre for Diffraction Data. Advances in X-ray Analysis, 48: 156-164.

Sauro, F.; De Waele, J.; Onac, B.P.; Galli, E.; Dublyansky, Y.; Baldoni, E. \& Sanna, L. (2014). Hypogenic speleogenesis in quartzite: The case of Corona 'e Sa Craba Cave (SWSardinia, Italy). Geomorphology, 211: 77-88. http://dx.doi.org/10.1016/j.geomorph.2013.12.031.

Showstack, R. (2011). Mars Opportunity rover finds gypsum veins. Eos, Transactions American Geophysical Unio, 92(51):479. http://dx.doi.org/10.1029/2011EO510003.

Squyres, S.W.; Grotzinger, J.P.; Arvidson, R.E.; Bell, J.F. 3rd; Calvin, W.; Christensen, P.R.; Clark, B.C.; Crisp, J.A.; Farrand, W.H.; Herkenhoff, K.E.; Johnson, J.R.; Klingelhöfer, G.; Knoll, A.H.; McLennan, S.M.; McSween, H.Y. Jr.; Morris, R.V.; Rice, J.W. Jr.; Rieder, R. \& Soderblom, L.A. (2004). In situ evidence for an ancient aqueous environment at Meridiani
Planum, Mars. Science, 306: 1709-1714. http://dx. doi.org/10.1126/science. 1104559 .

Szynkiewicz, A.; Ewing, R.C.; Moore, C.H.; Glamoclija, M.; Bustos, D. \& Pratt, L.M. (2010). Origin of terrestrial gypsum dunes-implications for Martian gypsum-rich dunes of Olimpia Undae. Geomorphology, 121: 69-83. http://dx.doi.org/10.1016/j.geomorph.2009.02.017.

Wilson, S.A. \& Bish, D.L. (2012). Stability of Mg - sulphate minerals in the presence of smectites: Possible mineralogical controls on $\mathrm{H} 2 \mathrm{O}$ and nutrient cycling on Mars. Proceeding of Australian Regolith and Clays Conference Mildura, 43-45.

Wiseman, S.M.; Arvidson, R.E.; Andrews-Hanna, J.C.; Clark, R.N.; Lanza, N.L.; Des Marais, D.; Marzo, G.A.; Morris, R.V.; Murchie, S.L.; Newsom, H.E.; Noe Dobrea, E.Z. \& Ollila, A.M. (2008). Phyllosilicate and sulfate-hematite deposits within Miyamoto crater in southern Sinus Meridiani, Mars. Geophysical Research Letters, 35: L19204. http://dx.doi. org/10.1029/2008GL035363.

Wray,J.J.;Squyres,S.W.;Roach,L.H.;Bishop,J.L.;Mustard, J.F. \& Noe Dobrea, E.Z. (2010). Identification of the Ca-sulfate bassanite in Mawrth Vallis, Mars. Icarus, 209:416-421.http://dx.doi.org/10.1016/j.icarus.2010. 06.001 .

Zhu, M.; Xie, H.; Guan, H. \& Smith, R.K. (2006). Mineral and lithologic mapping of Martian low-albedo regions using OMEGA data. 37th Lunar and Planetary Science Conference, 2173.

Zolotov, M.Y. \& Shock. E.L. (2005). Formation of jarosite-bearing deposits through aqueous oxidation of pyrite at Meridiani Planum, Mars, Geophysical Research Letters, 32: L21203. http://dx.doi. org/10.1029/2005GL024253. 